\title{
Effect of Disorder on Transport in Graphene
}

\author{
I. L. Aleiner ${ }^{1}$ and K. B. Efetov ${ }^{1,2,3}$ \\ ${ }^{1}$ Physics Department, Columbia University, New York, NY 1002\%, USA \\ ${ }^{2}$ Theoretische Physik III, Ruhr-Universität Bochum, 44780 Bochum, Germany \\ ${ }^{3}$ L. D. Landau Institute for Theoretical Physics, 117940 Moscow, Russia
}

(Dated: November 4, 2018)

\begin{abstract}
Quenched disorder in graphene is characterized by 5 constants and experiences the logarithmic renormalization even from the spatial scales smaller than the Fermi wavelength. We derive and solve renormalization group equations (RGEs) describing the system at such scales. At larger scales, we derive a non-linear supermatrix $\sigma$-model completely describing localization and crossovers between different ensembles. The parameters of this $\sigma$-model are determined by the solutions of the RGEs.
\end{abstract}

PACS numbers: 73.63.-b,81.05.Uw, 72.15.Rn

Introduction- Dirac spectrum of the quasiparticles in graphene confirmed by recent experiments $[1-3]$ is a consequence of the honeycomb lattice symmetry [4]. Although many properties of graphene can be understood in terms of ballistic motion of "relativistic" electrons described by a Dirac-like equation (see, e.g. [5, 6]), disorder plays an important role in sufficiently large samples.

Influence of disorder on a two-dimensional electron gases on the honeycomb lattice were studied in several works [7-13] within a self-consistent Born approximation (SCBA) standard for weakly disordered metals and superconductors [14]. Within SCBA, such quantities as density of states, or localization-less conductivity [7-10] were calculated. The weak localization (WL) correction was discussed in Ref. [12] and calculated in Ref. [13].

However, the SCBA is not justifiable for the Dirac spectrum, $|\varepsilon|=v|p|$, as it can be seen already in the fourth order perturbation theory in the disorder potential, see below, and a more careful analysis is needed.

In this paper, we reveal the origin of the logarithmic effects specific for the Dirac spectrum and different from WL. These corrections, see Fig. 1, are contributed by all spatial scales between the lattice constant $a$, and either wave-length $\lambda_{\varepsilon}=\hbar v / \varepsilon$, or the scale determined by disorder, and that is why we will coin the name of "ultraviolet logarithmic corrections" (UvLC) for them. We will sum up the leading series of UvLC within a one-loop RG. At larger linear scales all the physics is described by a non-linear $\sigma$-model[15] and UvLC enter as renormalized parameters in this model. We will show that the lowenergy asymptotics correspond to the orthogonal ensemble. Thus, the one-particle states are localized at any energies in contradiction with the findings of Ref. [1] of the minimal metallic conductivity in the undoped graphene.

Disordered Hamiltonian - In the undoped graphene two bands cross the Fermi level at $K$ and $K^{\prime}$ points. The corresponding Bloch functions comprise the basis of the four-dimensional (4d) representation of the planar group of the honeycomb lattice. We join them in a vector

$$
\vec{\varphi}^{T}(\mathbf{r})=\left(\left(\varphi_{A}, \varphi_{B}\right)_{A B} ;\left(\varphi_{B}^{*},-\varphi_{A}^{*}\right)_{A B}\right)_{K K^{\prime}}
$$

where we use the fact the points $K$ and $K^{\prime}$ are connected to each other by the time reversal symmetry

$$
\vec{\varphi}^{*}(\mathbf{r})=\hat{z} \vec{\varphi}(\mathbf{r}) ; \quad \hat{z} \equiv \tau_{y}^{A B} \otimes \tau_{y}^{K K^{\prime}} .
$$

where $4 \mathrm{~d}$-space of the wave-functions is represented as a direct product $A B \otimes K K^{\prime}$, of the sublattice $A B$ and "valley" $K K^{\prime} 2 \mathrm{~d}$-spaces, and $\tau_{x, y, z}^{\alpha}, \tau_{ \pm}^{\alpha}=\left(\tau_{x} \pm i \tau_{y}\right) / 2$ are the Pauli matrices acting in those spaces, $\alpha=A B, K K^{\prime}$ (we omit the physical spin).

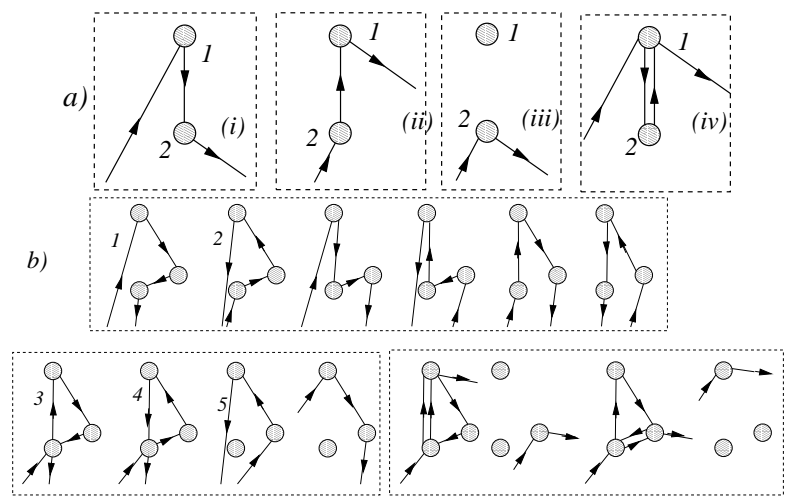

FIG. 1: Interfering scattering events involving (a) two- and (b) three-impurity scattering. They produce the correction (a) $\mathcal{O}\left[\ln \left(\lambda_{\varepsilon} / a_{0}\right)\right]$ and (b) $\mathcal{O}\left[\ln ^{2}\left(\lambda_{\varepsilon} / a_{0}\right)\right]$.

The low energy properties are described in $k \cdot \mathbf{p}$ approximation [4], i.e. the wave function $\Psi_{\varepsilon}(\mathbf{r})$ is looked for as $\Psi_{\varepsilon}(\mathbf{r})=\vec{\varphi}(\mathbf{r}) \cdot \vec{\Phi}_{\varepsilon}(\mathbf{r})$ with a smooth envelope, $\vec{\Phi}_{\varepsilon}(\mathbf{r})$, satisfying the effective Schrödinger equation $(\hbar=1)$

$$
\left(\hat{H}_{0}+\hat{V}(\mathbf{r})\right) \vec{\Phi}_{\varepsilon}(\mathbf{r})=\varepsilon \vec{\Phi}_{\varepsilon}(\mathbf{r}), \quad \hat{H}_{0}=-i v \overrightarrow{\hat{\Sigma}} \vec{\nabla}
$$

where $\vec{\nabla}=\left(\partial_{x}, \partial_{y}\right)$ and we introduced $4 \times 4$ matrices [16]

$$
\hat{\Sigma}_{x, y}=\mathbb{1}^{K K^{\prime}} \otimes \tau_{x, y}^{A B}, \quad \hat{G}_{m, i}=\tau_{m}^{K K^{\prime}} \otimes \tau_{i}^{A B} .
$$

For the spinless particles, the time-reversal symmetry (TRS) requires $\Psi^{*}(\mathbf{r})=\vec{\varphi}^{*}(\mathbf{r}) \cdot \vec{\Phi}^{*}(\mathbf{r})$ also to be the eigenstate of the original Hamitltonian. Together with Eq. (2) 
it constrains the effective Hamiltonian from Eq. (3) as

$$
\hat{H}_{0}=\hat{z} \hat{H}_{0}^{T} \hat{z}, \quad \hat{V}=\hat{z} \hat{V}^{T} \hat{z} .
$$

The disorder, $V(\mathbf{r})=V^{\dagger}(\mathbf{r})$, in Eq. (3) may break all the symmetries except of the TRS (5). This leads to

$$
\hat{V}(\mathbf{r})=\mathbb{1} u_{0}(\mathbf{r})+\hat{G}_{m, i} u_{m, i}(\mathbf{r}),
$$

where $u_{0}, u_{m, i}$ are real random functions, $\mathbb{1} \equiv \mathbb{1}^{A B} \otimes$ $\mathbb{1}^{K K^{\prime}}$, and the summation over indices $\{i, m\}=\{x, y, z\}$ is implied in Eqs. (6)-(7a). Equation (6) is the most general form for the disorder before averaging. Averaging must restore the rotation, reflection, $\left(C_{6 v}\right)$, and translation symmetries, which leads[17] to the most general Gaussian correlations $\left(\gamma_{0}>0, \Gamma_{m, i}>0\right)$

$$
\left\langle\hat{V}_{\mathbf{r}} \otimes \hat{V}_{\mathbf{r}}^{\prime}\right\rangle=\delta_{\mathbf{r}, \mathbf{r}^{\prime}}\left[\gamma_{0} \mathbb{1} \otimes \mathbb{1}+\Gamma_{m}^{i} \hat{G}_{m, i} \otimes \hat{G}_{m, i}\right] .
$$

Among the coefficients $\Gamma_{m, i}$ only four are independent:

$$
\Gamma_{z}^{z}=\gamma_{z}, \Gamma_{z}^{\{x, y\}}=\gamma_{\perp}, \Gamma_{\{x, y\}}^{z}=\beta_{z}, \Gamma_{\{x, y\}}^{\{x, y\}}=\beta_{\perp} .
$$

In what follows, we will treat the five independent parameters $\gamma, \beta$ of Eqs. (7) as the starting point of the theory, not trying to calculate their particular values determined by the details of the single impurity on the scale of the lattice constant. However, some qualitative conclusions can be drawn from the symmetry arguments. The diagonal term, $\left(\gamma_{0}>0, \Gamma_{m}^{i}=0\right)$, is generated by the dilute impurities such that a single impurity in the crystal still preserves the point symmetry $C_{6 v}$. Examples are the interstitial atom, or, more importantly, the remote charge impurity potential weakly varying on the scale of the lattice constant, $a$. On the other hand, a vacancy in otherwise perfect honeycomb lattice preserves only $C_{3 v}$ symmetry and allows for $\gamma_{z} \simeq \gamma_{0}$. The intravalley scattering, $\gamma_{\perp}>0$ arises e.g. due to the local deformation [18] or any bond disorder breaking the $C_{3}$ symmetry. The latter also causes the intervalley scattering, $\beta_{z, \perp}$. Finally, the terms of the similar structure are generated even by the multiple scattering by the pair of vacancies if the higher gradient terms are included in Hamiltonian (3).

Origin of $U v L C$ lies in the interference of the waves multiply scattered by the disorder potential, however, the configurations of the impurities giving rise to the effect differ from the familiar [15] WL. Thus, it is instructive to explain them, first, in terms of the counting of the scattering events and then present the rigorous calculation. It will also illuminate reasons for the failure of SCBA.

If there were no disorder potential the wave function $\vec{\Phi}_{\mathbf{k}}^{(0)}$ would be the plane-wave with with a momentum $\mathbf{k}$ and the structure of Eq. (1). Limit ourselves by positive energies $\varepsilon=v k, k \equiv|\mathbf{k}|$ for concreteness, and the projection operator $\hat{\mathbb{P}}(\mathbf{n}) \equiv(1+\boldsymbol{\Sigma} \mathbf{n}) / 2 ; \mathbf{n}=\mathbf{k} /|k|$ chooses the chirality corresponding to the positive energy. Consider an impurity placed at point $\mathbf{R}_{1}$. The asymptotics of wave function acquire the form $\vec{\Phi}_{\mathbf{k}}=\vec{\Phi}_{\mathbf{k}}^{(0)}+\vec{\Phi}_{\mathbf{k}}^{(1)}$, where

$$
\vec{\Phi}_{\mathbf{k}}^{(1)}= \begin{cases}\frac{\exp \left(\frac{i\left|\mathbf{r}_{1}\right|}{\lambda_{\varepsilon}}\right)}{\sqrt{-i\left|\mathbf{r}_{1}\right|}} \frac{\hat{\mathbb{P}}\left(\frac{\mathbf{r}_{1}}{\left|\mathbf{r}_{1}\right|}\right) \hat{f}_{1}}{\sqrt{2 \pi \lambda_{\varepsilon}}} \vec{\Phi}_{\mathbf{k}}^{(0)}\left(\mathbf{R}_{1}\right) ; & \frac{\left|r_{1}\right|}{\lambda_{\varepsilon}} \gg 1 ; \\ i\left(2 \pi\left|\mathbf{r}_{1}\right|^{2}\right)^{-1} \hat{\mathbf{\Sigma}} \mathbf{r}_{1} \hat{f}_{1} \vec{\Phi}_{\mathbf{k}}^{(0)}\left(\mathbf{R}_{1}\right) ; & \left|r_{1}\right| \ll \lambda_{\varepsilon}\end{cases}
$$

and $\mathbf{r}_{1} \equiv \mathbf{r}-\mathbf{R}_{1}$ is the distance from the impurity.

Equation (8) is valid for both $\left|\mathbf{r}_{1}\right|$ and $\lambda_{\varepsilon}$ much larger than the characteristic size of the scatterer. All the the details of the impurity are encoded in the matrix $\hat{f}_{1}$ which can be viewed as the scattering length, $a_{0}=\left\|\hat{f}_{1} \hat{f}_{1}^{\dagger}\right\|^{1 / 2}$. The value of $a_{0}$ is of the order of the size of the impurity which brings the estimate $a_{0} \simeq a$ for neutral impurities.

The asymptotic behavior of Eq. (8) at $\left|r_{1}\right| \gg \lambda_{\varepsilon}$ is nothing but the out-going spherical wave, corresponding to the $s$-scattering. It is important to emphasize that the dominance of the $s$-channel is the consequence of the large wave-length and not of the peculiarities of the impurity potential. The proportionality coefficient between the spherical wave and the amplitude of the plane-wave is the scattering amplitude that enables us to find the elastic scattering cross-section of the electron with the original momentum direction $\mathbf{n}_{i}$ to the final direction $\mathbf{n}_{f}$

$$
d \hat{s}=\frac{d \mathbf{n}_{f}}{2 \pi \lambda_{\varepsilon}}\left[\hat{\mathbb{P}}\left(\mathbf{n}_{f}\right) \hat{f}_{1} \hat{\mathbb{P}}\left(\mathbf{n}_{i}\right)\right] \otimes\left[\hat{\mathbb{P}}\left(\mathbf{n}_{f}\right) \hat{f}_{1} \hat{\mathbb{P}}\left(\mathbf{n}_{i}\right)\right]^{\dagger} .
$$

If there were no interference, Eq. (9) would describe all the kinetics of the system. For the impurity density, $n_{\text {imp }} \ll 1 / a_{0}^{2}$, the mean free path is estimated from

$$
\ell_{e l} \simeq\left(n_{i m p}\|\hat{s}\|\right)^{-1} \simeq \lambda_{\varepsilon} /\left(n_{i m p} a_{0}^{2}\right) \gg \lambda_{\varepsilon}, .
$$

To understand the role of the multiple scattering, consider the two impurity scattering, see Fig. 1a(i)-(ii).

Applying Eq. (8) twice we obtain for $\left|\mathbf{R}_{12}\right| \ll \lambda_{\varepsilon} \ll$ $\left|\mathbf{r}_{1}\right|$, the outgoing wave with $\hat{f}_{1} \rightarrow \delta \hat{f}=\hat{f}_{12}+\hat{f}_{21}$ and

$$
\hat{f}_{(i j)}=-\hat{f}_{i} \hat{\boldsymbol{\Sigma}}_{\mathbf{R}_{i j}} \hat{f}_{j}\left(2 \pi\left|\mathbf{R}_{i j}\right|^{2}\right)^{-1}, \mathbf{R}_{i j}=\mathbf{R}_{i}-\mathbf{R}_{j},
$$

for $i, j=1,2, i \neq j$, and $a_{0} \lesssim\left|\mathbf{R}_{12}\right| \lesssim \lambda_{\varepsilon}$. Equation (11) describes the two-impurity scattering amplitude for the given configuration of the impurities. The transport, see Eqs. (9)- (10), is determined by the powers of the $\hat{f}_{(i j)}$ averaged with respect to all configurations

$$
\begin{gathered}
\left\langle\hat{f}_{(i j)} \otimes \hat{f}_{(i j)}^{\dagger}\right\rangle=n_{i m p} \int \frac{d^{2} \mathbf{R}}{4 \pi^{2} R^{4}}\left[\hat{f}_{i} \hat{\boldsymbol{\Sigma}} \mathbf{R} \hat{f}_{j}\right] \otimes\left[\hat{f}_{j}^{\dagger} \hat{\mathbf{\Sigma}} \mathbf{R} \hat{f}_{i}^{\dagger}\right] \\
\simeq(4 \pi)^{-1} n_{i m p} \mathcal{L}\left[\hat{f}_{i} \hat{\Sigma}_{\alpha} \hat{f}_{j}\right] \otimes\left[\hat{f}_{j}^{\dagger} \hat{\Sigma}_{\alpha} \hat{f}_{i}^{\dagger}\right],
\end{gathered}
$$

where $\mathcal{L}=\ln \left(\lambda_{\varepsilon} / a\right)$. Analogously, we find

$$
4 \pi\left\langle\hat{f}_{(i j)} \otimes \hat{f}_{(j i)}^{\dagger}\right\rangle \simeq-n_{i m p} \mathcal{L}\left[\hat{f}_{i} \hat{\Sigma}_{\alpha} \hat{f}_{j}\right] \otimes\left[\hat{f}_{i}^{\dagger} \hat{\Sigma}_{\alpha} \hat{f}_{j}^{\dagger}\right] .
$$

Combining Eqs. (12a)-(12b) we obtain:

$$
4 \pi\left\langle\delta \hat{f} \otimes \delta \hat{f}^{\dagger}\right\rangle \simeq n_{i m p} \mathcal{L} d_{i j}^{i^{i^{\prime} j^{\prime}}}\left[\hat{f}_{i} \hat{\Sigma}_{\alpha} \hat{f}_{j}\right] \otimes\left[\hat{f}_{j^{\prime}}^{\dagger} \hat{\Sigma}_{\alpha} \hat{f}_{i^{\prime}}^{\dagger}\right],
$$


where non-vanishing coefficients are $d_{12}^{12}=d_{21}^{21}=1, d_{12}^{21}=$ $d_{21}^{12}=-1$, and summation over all repeating indices here as well as over $\alpha=x, y$ in Eqs. (12), (14) is implied.

Equation (12c) is the main result of the qualitative consideration revealing the origin of the logarithmic divergence. The Boltzmann kinetic equation systematically neglects those contributions. SCBA scheme accounts only for the diagonal components $d_{12}^{12}, d_{21}^{21}$ and misses all the other contributions. For the scalar disorder, e.g. it leads to the violating the TRS of the problem.

There are two more sources for the $n_{\text {imp }} a^{2} \mathcal{L}$ corrections. One of them is the correlation of the one-impurity scattering with the two-impurity scattering in which one of the impurities is visited twice as shown on Fig. 1a(iii)(iv). The corresponding result is easily obtained from the three-impurity scattering amplitude [cf, Eq. (11)]

$$
\hat{f}_{(i j k)}=\left(\hat{f}_{i} \hat{\boldsymbol{\Sigma}} \mathbf{R}_{i j} \hat{f}_{j} \hat{\boldsymbol{\Sigma}} \mathbf{R}_{j k} \hat{f}_{k}\right) /\left(4 \pi^{2}\left|\mathbf{R}_{i j}\right|^{2}\left|\mathbf{R}_{j k}\right|^{2}\right),
$$

and we find

$$
4 \pi\left\langle\hat{f}_{(121)} \hat{f}_{2}^{\dagger}\right\rangle \simeq n_{i m p} \mathcal{L}\left[\hat{f}_{1} \hat{\Sigma}_{\alpha} \hat{f}_{2} \hat{\Sigma}_{\alpha} \hat{f}_{1}\right] \otimes \hat{f}_{2}^{\dagger} .
$$

This correction is missing in SCBA. The last logarithmic effect arising in this order is the logarithmic dependence $\propto n_{\text {imp }} a^{2} \varepsilon \ln \varepsilon$ of the averaged forward scattering amplitude. It does not affect scattering processes directly but renormalizes the spectrum of the free Hamiltonian $H_{0}$.

Analogously, one considers the scattering from the three-impurity configurations, see Fig. 1b), starting from the expression (13) and finds 54 contributions $\propto n_{\text {imp }}^{2} \mathcal{L}^{2}$ only 6 of which are included in the SCBA.

Interference of few processes, is somewhat special as they do not vanish completely even if the distance between impurities is larger than $\lambda_{\varepsilon}$. In fact the interference $1-2$ and $3-5$ are the first contributions giving rise to the WL correction which is also logarithmic. The WL, however, originates from the spatial scales larger than $\ell_{e l}$, and that is why they can be separated from the UvLC.

Field theory and $R G$ - Let us turn to the rigorous calculations using the supersymmetry method. Due to the $4 \times 4$ matrix structure of the Hamiltonian, Eqs. $(3,6)$, the supervectors $\psi$ should have 4 times more components than usually used[15], i.e we need 32-components for calculation of the conductivity. The resulting $32 \mathrm{~d}-$ space can be presented as a direct product of five $2 \mathrm{~d}$ ones, $A B \otimes K K^{\prime} \otimes A R \otimes e h \otimes g$, where $A R, e h$ and $g$ are the retarded-advanced, particle-hole, and the fermion-boson sectors. Averaging over $\hat{V}$ and using Eq. (7a), we find

$$
\begin{aligned}
& \langle\ldots\rangle=\int \cdots \exp (-L[\psi]) \mathcal{D} \psi, \psi^{\dagger} \hat{\boldsymbol{\Lambda}}=\bar{\psi}=[\hat{\mathbb{C}} \psi]^{T}, \\
& \hat{\boldsymbol{\Lambda}}=\hat{\Lambda} \otimes \mathbb{1}^{A B} ; \hat{\Lambda}=\tau_{z}^{A R} \otimes \mathbb{1}^{K K^{\prime}} \otimes \mathbb{1}^{e h} \otimes \mathbb{1}^{g} ; \quad(15) \\
& \hat{\mathbb{C}}=\hat{C} \otimes \tau_{y}^{A B}=\hat{z} \otimes \mathbb{1}^{A R} \otimes\left(\tau_{-}^{e h} \otimes \mathbb{1}^{g}-\tau_{+}^{e h} \otimes \tau_{z}^{g}\right) .
\end{aligned}
$$

where $\ldots$ on the LHS stand for any combination of advanced/retarded Green functions $\hat{G}^{A, R}=$ $\left(\varepsilon \mp \omega / 2-\hat{H}_{0}-\hat{V} \mp i 0\right)^{-1}$ and $\cdots$ in the RHS for the corresponding sources breaking the supersymmetry. Their form can be found in Ref. [15] but it will not be important for us. The Lagrangian $L[\psi]=L_{0}[\psi]+L_{\text {int }}[\psi]$ is given by (sum over $i, m=x, y, z$ is implied)

$$
\begin{aligned}
& L_{0}[\psi]=i \int \bar{\psi}\left[\varepsilon-\hat{\mathbb{H}}_{0}-\hat{\boldsymbol{\Lambda}}\left(\frac{\omega}{2}+i 0\right)\right] \psi d \mathbf{r}, \\
& L_{\text {int }}[\psi]=\frac{1}{2} \int\left[\gamma_{0}(\bar{\psi} \psi)^{2}+\Gamma_{m}^{i}\left(\bar{\psi} \hat{\mathbb{G}}_{m, i} \psi\right)^{2}\right] d \mathbf{r}
\end{aligned}
$$

where $\left(\hat{\mathbb{H}}_{0}, \hat{\mathbb{G}}_{m, i}\right)=\left(\hat{H}_{0}, \hat{G}_{m, i}\right) \otimes \mathbb{1}^{g} \otimes \mathbb{1}^{A R} \otimes \mathbb{1}^{e h}$.

The perturbation theory in $L_{i n t}[\psi]$ leads to UvLC and we can calculate the integral (15) using a RG scheme. We decompose $\psi$ as $\psi=\psi_{0}+\tilde{\psi}$, where $\tilde{\psi}$ is slow and $\psi_{0}$ is fast, and integrate out $\psi_{0}$. We rescale $\tilde{\psi}$ and the coordinates to keep the coefficient in front of $\varepsilon$ and the ultraviolet cut-off intact. It gives back Eq. (16), with renormalized couplings $\gamma_{0}, \Gamma_{m}^{i}$, Eq. (7b) and the velocity $v$, Eq. (3). This yields RGE (see Ref. [17] for details), which we display for the most interesting case $\gamma_{0} \gtrsim \Gamma_{m}^{i}$ :

$$
\begin{aligned}
& 2 \pi v \partial_{t} v=-\left(\gamma_{0}+g_{\|}+2 g_{\perp}\right) ; \\
& 9 \pi v^{2} \partial_{t} \gamma_{0} \approx 2\left(g_{\|}^{2}+2 g_{\perp}^{2}\right) ; \pi v^{2} \partial_{t} \delta g_{\|, \perp} \approx-3 \gamma_{0} \delta g_{\|, \perp} ; \\
& 9 \pi v^{2} \partial_{t} g_{\|} \approx-8 g_{\|}^{2}-20 g_{\|} g_{\perp}+14 g_{\perp}^{2} ; \\
& 9 \pi v^{2} \partial_{t} g_{\perp} \approx 4 g_{\|} g_{\perp}-18 g_{\perp}^{2} .
\end{aligned}
$$

Here $g_{\|}=\gamma_{z}+2 \gamma_{\perp} ; g_{\perp}=\beta_{z}+2 \beta_{\perp} ; \delta g_{\|}=\gamma_{z}-\gamma_{\perp}$; $\delta g_{\perp}=\beta_{z}-\beta_{\perp} ; t$ is the logarithm of the running energy.

Solving Eqs. (17) down to the energy, $|\varepsilon|$, we find

$$
v(\varepsilon)=\left(\frac{\gamma_{0}}{\pi} \ln \frac{|\varepsilon|}{\varepsilon_{0}}\right)^{1 / 2}, \quad \gamma_{0}(\varepsilon)=\gamma_{0}+\mathcal{O}\left(\frac{g_{\|}(\varepsilon)}{\gamma_{0}}\right),
$$

where $\varepsilon_{0} \simeq J \exp \left(-\pi v_{0}^{2} / \gamma_{0}\right)$ is the energy at which the first loop RG breaks down, and $J$ is the bandwidth.

The last of Eqs. (17) yields non-mixed valleys, $g_{\perp}=0$, to be unstable, and $g_{\perp}$ flows towards

$$
g_{\|}(\varepsilon) \approx g_{\perp}(\varepsilon) \approx 9 \gamma_{0} /\left\{14 \ln \left[t^{*} / \ln |\varepsilon| / \varepsilon_{0}\right]\right\},
$$

$\delta g_{\|, \perp}(\varepsilon) \propto \ln ^{3}\left(|\varepsilon| / \varepsilon_{0}\right)$, and $t^{*}$ depends on $g_{\|, \perp}(\varepsilon \simeq J)$.

Equations (18) enables one to use the renormalized parameters for the standard calculation of the transport coefficients. The diffusion coefficient $D(\varepsilon)$ is given by

$$
D(\varepsilon)=v^{2}(\varepsilon) \tau_{t r}(\varepsilon) / 2 ; 1 / \tau_{t r}(\varepsilon)=\pi \gamma_{0} \nu_{\varepsilon} / 4,
$$

where $\nu_{\varepsilon}=|\varepsilon| /\left(\pi v_{\varepsilon}^{2}\right)$ is the density of states (per one physical spin). Einstein relation and Eqs. (18) yield [20]

$$
\sigma=\frac{4 e^{2}}{\pi^{2} \hbar} \ln \left(\frac{|\varepsilon|}{\varepsilon_{0}}\right) .
$$

Equation (21) is the universal formula for the UvLC. It describes either the temperature or the density dependence of the conductivity limited by the short-range 
disordered potential $|\varepsilon| \rightarrow \max \left(\varepsilon_{F}, T\right)$. It also gives the leading dependence of the thermopower through the Mott-Cutler formula. At $|\varepsilon| \lesssim \varepsilon_{0}$, the logarithms are cut by $1 / \tau_{t r}$, which leads to the replacement of $\ln (\cdot)$ to the factor of the order of unity. The precise value of this factor, however, can not be calculated within Eqs. (17).

Nl $\sigma$-model and localization- At distances larger than $v / \max \left(|\varepsilon|, \varepsilon_{0}\right)$ soft modes giving rise to UvLC freeze out, and only the degrees of freedom guarded by the fundamental symmetries of the system remain gapless. All those degrees of freedom are described by $n l \sigma$-model [15] and functional integral (15) is replaced by integral over $16 \times 16$ supermatrices in $K K^{\prime} \otimes A R \otimes e h \otimes g$ space [17]:

$$
\begin{aligned}
& \langle\ldots\rangle=\int \cdots \exp (-F[\hat{Q}]) \mathcal{D} \hat{Q}, \hat{Q}^{2}=\mathbb{1} ; \hat{Q}=\hat{C} \hat{Q}^{T} \hat{C}^{T} ; \\
& \hat{Q}^{\dagger}=\hat{\mathbb{K}} \hat{Q} \hat{\mathbb{K}} ; \quad \hat{\mathbb{K}}=\left(\begin{array}{cc}
\mathbb{1}^{g} & 0 \\
0 & \tau_{3}^{g}
\end{array}\right)_{A R} \otimes \mathbb{1}^{K K^{\prime} \otimes \mathbb{1}^{e h} .}
\end{aligned}
$$

The free energy functional $F[Q]$ takes the form

$$
\begin{aligned}
& F=\frac{\pi \nu_{\varepsilon}}{16} \operatorname{Str} \int\left\{D(\varepsilon)\left(\nabla \hat{Q}-\frac{i e \boldsymbol{A}}{c}\left[\hat{Q} ; \hat{\mathcal{T}}_{3}\right]\right)^{2}+2 i \omega \hat{\Lambda} \hat{Q}\right. \\
& \left.-\frac{\pi \nu_{\varepsilon} g_{\|}}{4}\left[\hat{\rho}_{z}, \hat{Q}\right]^{2}-\frac{\pi \nu_{\varepsilon} g_{\perp}}{4}\left(\left[\hat{\rho}_{x}, \hat{Q}\right]^{2}+\left[\hat{\rho}_{y}, \hat{Q}\right]^{2}\right)\right\} d \mathbf{r} \\
& \left(\hat{\rho}_{\alpha}, \hat{\mathcal{T}}_{3}\right)=\left(\tau_{\alpha}^{K K^{\prime}} \otimes \mathbb{1}^{e h}, \mathbb{1}^{K K^{\prime}} \otimes \tau_{z}^{e h}\right) \otimes \mathbb{1}^{A R} \otimes \mathbb{1}^{g},
\end{aligned}
$$

where $\boldsymbol{A}$ is the vector potential due to the magnetic field normal to the graphene, and the entries include UvLC.

Equation (23) is the only form allowed by the symmetries of the problem. The symmetries of the $Q$-matrices, Eq. (22), correspond to the two replicas of symplectic ensemble, which would flow to the limit of large conductances. However, due to $g_{\|, \perp}>0$ only $\hat{Q} \propto \mathbb{1}^{K K^{\prime}}$ is allowed at large distances and one obtains a generic orthogonal ensemble. Thus, all the eigenstates are localized. Schematic temperature dependence of $\sigma$ for undoped graphene is sketched on Fig. 2, [22].

The fist loop correction in Eq. (23) yields the WL [20]

$$
\begin{aligned}
& \Delta \sigma_{W L}=\frac{e^{2}}{2 \pi^{2} \hbar} \sum_{j=0}^{3} d_{j}\left[\ln \left(\frac{1}{-i \omega_{j} \tau_{t r}}\right)-Y\left(\frac{1}{-i \omega_{j} \tau_{B}}\right)\right] \\
& \omega_{0}=\omega+i 0 ; \omega_{1}=\omega+\frac{i}{\tau_{\perp}} ; \omega_{2,3}=\omega+\frac{i}{2 \tau_{\|}}+\frac{i}{2 \tau_{\perp}},
\end{aligned}
$$

where $-d_{0}=d_{1,2,3}=1, \tau_{\|, \perp}^{-1}=2 \pi \nu_{\varepsilon} g_{\|, \perp}, \tau_{B}^{-1}=$ $4 D(\varepsilon) e B /(\hbar c), Y(x)=\psi(x+1 / 2)+\ln x$ and $\psi(x)$ is the di-gamma function. Inelastic processes are accounted for by $-i \omega \rightarrow-i \omega+\tau_{\phi}^{-1}$, where $\tau_{\phi}$ is the dephasing time.

Equation (24) agrees with Ref. [13]. The new information here is the logarithmic dependence of the parameters on the electron energy [21], see Eqs. (18)-(20).

In conclusion, we have presented a complete description of a disordered graphene and demonstrated that

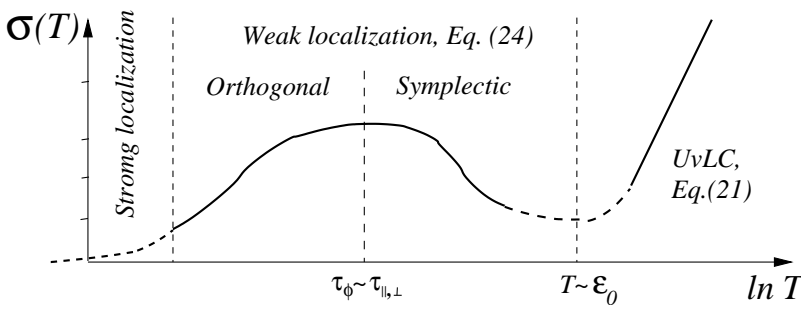

FIG. 2: Schematic dependence, $\sigma(T)$, for the undoped graphene and for $\tau_{\phi}^{-1} \propto T$.

there are two different types of logarithmic contributions into physical quantities, see Eqs. (21) and (24).

We are grateful to V. Falko, L. Glazman and P. Kim for remarks and to Transregio 12 for a support of K.B.E.

[1] K.S. Novoselov et al, Science 306, 666 (2004); Nature 438, 197 (2005); Nature Physics 2, 177 (2006).

[2] Y. Zhang et al, Phys. Rev. Lett. 94, 176803 (2005); Y. Zhang et al, Nature 438, 201 (2005)

[3] C. Berger et al, J. Phys. Chem. B 10819912 (2004); Science 312, 1191 (2006); J.S. Bunch et al, Nano Lett. 5, 287 (2005);

[4] For a general analysis, see G.L. Bir and G.E. Pikus, Symmetry and strain-induced effects in semiconductors, New York, Wiley (1974).

[5] V.P. Gusynin and S.G. Sharapov, Phys. Rev. Lett. 95, 146801 (2005); D. A. Abanin, P.A. Lee, L.S. Levitov, conmat/0602645; J. Tworzydlo et.al. ; cond-mat/0603315; V.V. Cheianov and V.I. Falko, cond-mat/ 0603624.

[6] M.I. Katsnelson, cond-mat/0512337; cond-mat/0606611.

[7] N.H. Shon, T. Ando, J. Phys. Soc. Jpn 67, 2421 (1998)

[8] H. Suzuura, T. Ando, Phys. Rev. Lett. 89, 266603 (2002)

[9] Y. Zheng and T. Ando, Phys. Rev. B 65, 245420 (2002)

[10] T. Ando, Y. Zheng, and H. Suzuura, J. Phys. Soc. JPN. 71, 1318 (2002)

[11] N.M.R. Peres, F. Guinea, and A.H. Castro Neto, Phys. Rev. B 73, 125411 (2006)

[12] A.F. Morpurgo and F. Guinea, cond-mat/0603789.

[13] E. McCann et al, cond-mat/0604015

[14] A.A. Abrikosov, L.P. Gorkov, and I.E. Dzyaloshinskii, Methods of Quantum Field Theory in Statistical Physics, Prentice Hall, New York (1963)

[15] K.B. Efetov, Supersymmetry in Disorder and Chaos, Cambridge University Press, New York (1997).

[16] The matrices $\hat{\Sigma}, \hat{z}$ are different from those of Refs. [7, 8, 11, 13], due to the different choice of the vector in Eq. (1).

[17] I.L. Aleiner, K.B. Efetov (in preparation).

[18] S.V. Iordanskii and A.E. Koshelev, JETP Lett, 41, 574 (1985).

[19] S.V. Morozov et al, cond-mat/0603826.

[20] Here, we restored $\hbar$ and the spin degeneracy.

[21] Warping terms considered in Ref. [13] can be shown to produce $\tau_{\perp} / \tau_{w} \propto \varepsilon^{2} \ln \left(|\varepsilon| / \varepsilon_{0}\right) \rightarrow 0, \varepsilon \rightarrow 0$.

[22] Nomura and MacDonald, cond-mat/0606589, claimed $\sigma \simeq 4 e^{2} / h$ at $\varepsilon \rightarrow 0$. We believe this conclusion to stem from the incorrect form of the disordered Hamiltonian. 